\title{
Peer Reviewers for the Journal of the American Board of Family Medicine in 2008
}

External peer reviewers are the unsung heroes of the $7 A B F M$. Here we acknowledge and thank all who so generously gave their time and expertise to build the evidence base of family medicine, as well as to advance the scholarship of their peers. We also take this opportunity to identify how our peer review processes are aligned with the recent $\mathrm{Na}$ tional Institutes of Health (NIH) plans to enhance its peer-review system.

In September, 2008, the NIH Peer Review Oversight Committee announced plans to enhance NIH peer-review activity, stating, "The excellence of peer review is directly correlated to our ability to recruit and retain the most accomplished, broad-thinking, and creative scientists to serve on study sections" (available at http://enhancing-peer-review.nih.gov). Although the NIH peer review enhancements are specific to the federal grant review process, we found that those related to "engaging the best reviewers" are also pertinent to our efforts at the $7 A B F M$. These are excerpted below along with comments specifying how the $7 A B F M$ addresses these best practices.

\section{NIH: "Recruit the Best Reviewers"}

FABFM alignment: We aim to secure a content specialist and family physician generalist for each reviewed manuscript to provide a broad and scholarly critique to assist authors in disseminating their work. In 2008, reviews were submitted from 43 states and 21 countries.

\section{NIH: "Improve Reviewer Retention"}

$\mathcal{F A B F M}$ alignment: We recognize that reviewers are very busy, and we avoid overburdening our reviewers with many requests. To expand our peer reviewer database, we actively recruit new reviewers with most new submissions; authors are also invited to suggest reviewers.

\section{NIH: "Provide Benefits for Reviewers"}

FABFM alignment: Reviewers receive a copy of the editor's decision letter that includes the reviews for the manuscripts they critiqued. In 2008, we instituted an annual letter to those reviewers who provide exceptional service to the $7 A B F M$. With this we intend to show our appreciation and strengthen relations with the family medicine research community. We also consider requests from excellent reviewers for letters of support for promotion or tenure.

\section{NIH: "Enhance Reviewer Training"}

fABFM alignment: We provide resources for peer reviewers, which are available in the "Peer Reviewers" area on the fournal's home page (www.jabfm.org). These include an article about how to review a manuscript, ${ }^{1}$ an article about the $7 A B F M$ peer-review process, ${ }^{2}$ and an article about what we seek in reviewer critiques. ${ }^{3}$ The editors also provide occasional workshops on journal peer reviewing at national primary care. Reviewers should also be aware of the $\mathcal{F} A B F M$ editorial statement regarding conflict of interest. ${ }^{4}$

\section{NIH: "Allow Flexibility"}

$\mathcal{F A B F M}$ alignment: Reviewers are encouraged to enter into their Rapid Review reviewer profile dates in which they will be unavailable for requests for manuscript review. Reviewers who receive a request that they are unable to accept are encouraged to let us know of such circumstances; some reviewers take this opportunity to suggest alternative potential reviewers.

\section{NIH: "Streamline Applications"}

fABFM alignment: All manuscript submissions are assigned to an editor for internal review and a decision to send the paper out for external peer review. ${ }^{2}$ In 2008, 26\% of unsolicited manuscripts were rejected after this internal review. The top reasons for rejection (internal review only and external review combined) were because it added little new information to topic (34\%); it was faulty research (26\%); or it was poorly written $(18 \%)$.

\section{Electronic Table of Contents Alerts}

We invite our peer reviewers to submit their best work to the $7 A B F M$. We are an open-access journal 
with a wide readership. At the end of 2008, 67,015 members of the primary care community received our electronic Table of Contents, which is sent when each issue posts online. These eTOCs have hyperlinks to each new article. Sign up for eTOCS at http://www.jabfm.org/subscriptions/etoc.shtml.

\section{Recruiting New Peer Reviewers}

New reviewers are welcome; researchers, faculty, and community-based physicians are invited to enlist as peer reviewers for the $7 A B F M$ (see the peer

Ya'aqov M. Abrams

John Abramson

David A. Acosta

David C. Agerter

Erin J. Aiello

James E. Aikens

Grace A. Alfonsi

Deborah Allen

William A. Alto

Gail M. Amundson

J. Ellen Anderson

Ronald J. Angel

Jack Ansell

Christine Arenson

Cheryl B. Aspy

Liv Berit Augestad

John Bachman

Elisabeth L. Backer

Eric W. Bakker

Beth Barnet

Kirsten Barrett

Monina Bartoces

Kay A. Bauman

Max Bayard

Elizabeth A. Bayliss

Oralia V. Bazaldua

Howard B. Beckman

Marvin Moe Bell
Franco Benazzi

Michal Benderly

Ian M. Bennett

Alfred O. Berg

Stephen Berman

Frances E. Biagioli

Dawn Bielawski

Juliann Binienda

Helen J. Binns

B. Wayne Blount

Alan Blum

Thomas Bodenheimer

Patrik Borg

Hayden B. Bosworth

Rachel Bramson

Kent Bream

Patrick Bridge

Howard Brody

Carlos Brotons

David W. Brown

Richard L. Brown

Sharon A. Brown

Cynthia M. Bulik

Sandra K. Burge

Lucinda M. Buys

Suzanne M. Cadarette

Peter J. Carek

Nancy Carter reviewer registration form on the website at www.jabfm.org).

\section{Reviewer Performance}

In 2008, 390 individuals submitted to the $7 A B F M$ editors 455 peer reviews and advice regarding suitability for publication. Reviewers took an average of 24 days (median, 25) to submit a review. Thanks to the following reviewers for their outstanding responsiveness:
Ashwin Chatwani

Rajesh Chauhan

Frederick M. Chen

John A. Chiladakis

Kaj S. Christensen

Richard D. Clover

Andreas Cohrssen

Vilma Cokkinides

Yeates Conwell

Michael A. Crouch

Rebecca Crowell

Brian K. Crownover

Richard W. Crummer

Jennifer F. Culhane

Larry Culpepper

William Curry

Craig W. Czarsty

Timothy P. Daaleman

Peter G. Danis III

Robert J. Darios

Martin G. Dawes

Cheryl Dee

M. Dianne Delva

Raymond Y. Demers

Cristina Demian-Popescu

Richard A. Deyo

Joel Dickerman

L. Miriam Dickinson 
Allen J. Dietrich

Joseph R. DiFranza

Martey S. Dodoo

Lisa Dodson

Rowena J. Dolor

Steven A. Dosh

Tanya Dougherty

Susan Dovey

Benjamin Druss

Anne L. Dunlop

Eileen F. Dunne

Scott Eathorne

Charles B. Eaton

Nancy C. Elder

William G. Elder, Jr.

Eva K. Ellegard

William A. Ellert

John W. Ely

Charles C. Engel

John W. Epling, Jr.

Edzard Ernst

Paolo Fabbri

Krista Farey

Adrianne Feldstein

Joshua J. Fenton

Robert L. Ferrer

Karl B. Fields

Edgar Figueroa

Kenneth S. Fink

Murray M. Finkelstein

Kevin Fiscella

Colleen T. Fogarty

Patricia Fontaine

Stuart Forman

E. Foster

Arthur Fournier

Chester H. Fox
Erica Frank

Jennifer Frank

Peter Franks

Michael D. Freeman

Linda M. French

Ivar L. Frithsen

Vicki Fung

Sim S. Galazka

Mauro Gallitelli

Joseph J. Gallo

Elizabeth Garrett

James M. Gill

Dwenda K. Gjerdingen

Stephen Goggans

Marji Gold

Adam O. Goldstein

Rise B. Goldstein

Jodi M. Gonzalez

Robert Graham

Judith A. Gravdal

Beverly B. Green

Larry A. Green

Michael E. Green

Tomas L. Griebling

Kim Griswold

Michael Grover

Jasen W. Gundersen

Warren G. Guntheroth

A. Gutke

David L. Hahn

S. A. Haje

Jennifer L. Hamilton

E. Haneke

Susan Harris

Kelly Haskard

Robert L. Hatch

Diana L. Heiman
Robin Helm

Eric Henley

Joshua A. Hirsch

Joseph Hobbs

Michael F. Holick

Christina T. Holt

Eugene Hong

Phyllis Horns

Margaret S. Houston

Sharon K. Hull

Jon Eric Isaacson

Livia Jackson

Carlos Roberto Jaen

Paul A. James

Sandeep Jauhar

Shannon B. Jenkins

Sue Jessop

Raymond Jian

Masahito Jimbo

Michel R. Joffres

David W. Johnson

Mark S. Johnson

Jason D. Jones

Nikolaos V. Kafkas

Jordan F. Karp

Robert D. Keeley

Amy J. Keenum

Omar A. Khan

Niharika Khanna

George E. Kikano

Colin P. Kopes-Kerr

M. Z. Koubeissi

Grace M. Kuo

Jesse Lachter

Joseph M. Lane

Robert Langan

Lixing Lao 
Cristina Lara-Castro

Frank Lawler

Edward W. Lee

Lawrence M. Leeman

Patricia M. Lenahan

Stephen Lerner

Barcey T. Levy

Joseph A. Lieberman III

Elizabeth Lin

Maja-Lisa Loechen

Everett E. Logue

Kate Lorig

David P. Losh

Margaret M. Love

Bing Lu

Todd Lucas

Jon Lurie

Catherine L. Lysack

Paula K. Maasilta

Diane J. Madlon-Kay

Eli Magen

Michael K. Magill

Margaret A. Maglione

Mark Mahowald

Kamal A. Mansour

Jun J. Mao

Katherine Margo

Cara Marshall

Luca Mascitelli

Dimitrios K. Matthaiou

Charles Maynard

Gary McCord

S. E. McGregor

Helen E. McIlvain

W. E. Mehling

David R. Mehr

Joel H. Merenstein
J. L. Michener

Daniel Miller

Sandra Miller

Arunima Misra

James W. Mold

Alain J Montegut

Michael V. Moore

Pablo A. Mora

Karen Muchowski

Paul S. Mueller

Marlon P. Mundt

Joseph M. Murley

Zsolt J. Nagykaldi

Rajasree J. Nair

Jatinder Narang

Laeth S. Nasir

Donald E. Nease, Jr.

Eddie Needham

Jon O. Neher

Richard Neill

Lynne S. Nemeth

Herbert B. Newton

Warren P. Newton

Giang T. Nguyen

Polly H. Noel

Thomas E. Norris

Mary Patricia Nowalk

Jim Nuovo

Steven M. Ornstein

Lynda J. Owen

Wilson D. Pace

J. F. Pagel

Heather Paladine

Scott A. Paluska

Michael K. Park

Bennett Parnes

Donald Pathman
Valory N. Pavlik

Kevin A. Pearce

Randolph L. Pearson

William S. Pearson

Wayne F. Peate

Michael J. Petrizzi

T. Grant Phillips II

William R. Phillips

Marty S. Player

John H. Porcerelli

David Price

Michael D. Prislin

Elizabeth Proude

Jacob P. Prunuske

James C. Puffer

Perry A. Pugno

Wayne Putnam

Parvez A. Qureshi

David Rabago

Howard K. Rabinowitz

Kalyanakrishnan Ramakrishnan

Jo Marie Reilly

Michael D. Reis

Erika Ringdahl

Louis Ritz

Peter A. Rives

Charo Rodriguez

Hector P. Rodriguez

James E. Rohrer

Michael P. Rosenthal

Michael G. Ross

Mack T. Ruffin IV

R. Ruiz-Moral

Monika M. Safford

George Samraj

Haleh Sangi-Haghpeykar

John W. Saultz 
Judith A. Savageau

Ramesh Saxena

Ellen I. Schafheutle

Robert M. Schainfeld

Maryjean Schenk

Helen Schneider

Kendra Schwartz

Thomas L. Schwenk

Jeffrey B. Schwimmer

John G. Scott

Dean A. Seehusen

Peter A. Selwyn

Eric K. Shaw

Jun Shu

Leonard Allen Shulman

Pesach Shvartzman

Ajai Singh

Paul A. Sloan

L. F. P. Smith

Peter C. Smith

Douglas Smucker

Leif I. Solberg

Adam J. Sorscher

Stephen J. Spann

Stuart M. Speedie

Joseph B. Stanford

Jamie Stang

Barbara Starfield

Michael Stehney
Phyllis K. Stein

Nancy G. Stevens

Stefan Stoerk

Gregg D. Stoner

Mark L. Stovak

Joseph Straton

Jeff L. Susman

Andrew L. Sussman

J. Swan

Alfred F. Tallia

Peter G. Teichman

Kay Tetzlaff

Clayton L. Thomason

Dixie L. Thompson

G. A. Tommaselli

John M. Tovar

Matthew E. Ulven

Jose M. Valderas

Ron Vallario

Daniel J. Van Durme

Richard D. Vann

Steven D. Vannoy

Anthony J. Viera

Viju Vijaysadan

Daniel C. Vinson

Ilia D. Volkov

Peggy J. Wagner

Gordon Walbroehl

Peter D. Watson
Ganesa Wegienka

Barry D. Weiss

Myrna Weissman

Frances K. Wen

James J. Werner

Aaron M. White

Philip S. Whitecar

Wilhelmine Wiese-Rometsch

Jennifer E. Wildes

Thad E. Wilson

Daniel K. Winstead

Walter P. Wodchis

Leslie E. Wolf

William M. Woodhouse

Scott E. Woods

W. Michael Woods

Jinping $\mathrm{Xu}$

K. T. Xu

Bektas Murat Yalcin

Barbara P. Yawn

Rodney B. Young

Rosalie F. Young

Philip Zazove

Therese Zink

Adam J. Zolotor

Stephen J. Zyzanski

Anne Victoria Neale, $\mathrm{PhD}, \mathrm{MPH}$

Marjorie A. Bowman, MD, MPA

Phillip Lupo, MLIS

\section{References}

1. Roberts LW, Coverdale J, Edenharder K, Louie A. How to review a manuscript: a "down-to-earth" approach. Acad Psychiatry 2004;28:81-7. Available at http://ap.psychiatryonline.org/cgi/content/full/28/2/ 81. Accessed 9 January 2009.

2. Neale AV, Bowman, MA. Peer review process of the Fournal of the American Board of Family Medicine. J Am Board Fam Med 2006;19:209-10. Available at http:// www.jabfm.org/cgi/content/full/19/2/209. Accessed 9 January 2009.
3. Neale AV, Schwartz KL, Bowman, MA. Peer reviewing for the Fournal of the American Board of Family Medicine: what does it take? J Am Board Fam Med 2006;19:643-647. Available at: http://www.jabfm. org/cgi/reprint/19/6/643.pdf. Accessed 9 January 2009.

4. Neale AV, Schwartz KL, Bowman MA. Conflict of interest: can we minimize its influence in the biomedical literature? J Am Board Fam Med 2005;18:411-3. Available at http://www.jabfm.org/cgi/reprint/18/5/ 411.pdf. Accessed 9 January 2009. 